\title{
Association between neutrophilic granulocyte percentage and depression in hospitalized patients with heart failure
}

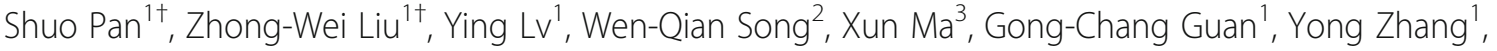
Shun-Ming Zhu', Fu-Qiang Liu', Bo Liu', Zhi-Guo Tang ${ }^{1}$ and Jun-Kui Wang ${ }^{1 *}$

\begin{abstract}
Background: Previous researches reveal that depression is associated with increased inflammatory markers. As a simple and cheap inflammatory marker, we hypothesize that neutrophilic granulocyte percentage is associated with depression in hospitalized heart failure patients, whose prevalence of depression is at a very high level.

Methods: Three hundred sixty-six cases of hospitalized heart failure patients with left ventricular ejection fraction $($ LVEF) $\leq 45 \%$ and New York Heart Association (NYHA) class II-IV were enrolled. All the enrolled patients received Hamilton Rating Scale for Depression (24-items) (HAM-D 24 ). The demographic, clinical data, blood samples and echocardiography were documented. The Pearson simple linear correlation was performed to evaluate the confounding factors correlated with $\mathrm{HAM}-\mathrm{D}_{24}$ depression index. The significantly correlated factors were enrolled as independent variables in Logistic regression to determine the risk or protective factors for depression, which was taken as dependent variable.

Results: Two hundred ten cases of hospitalized heart failure patients (57.4\%) had depression. Among them, 134 patients (63.8\%) had mild depression, 58 patients (27.6\%) had moderate depression and 18 patients (8.6\%) had severe depression. Pearson simple linear correlation revealed that in hospitalized patients with heart failure, the neutrophils granulocyte percentage was positively correlated with the HAM-D ${ }_{24}$ depression index $(r=.435$, $p<.001)$. After the adjustment of age, BMI, number of members of the household, smoking index, New York Heart Association (NYHA) classification, hemoglobin, TC, LDL-C, creatinine, cystatin-C, TBIL and albumin, the neutrophils granulocyte percentage is still significantly associated with depression in hospitalized heart failure patients $(O R=1.046, p<.001)$.
\end{abstract}

Conclusions: The neutrophils granulocyte percentage may be used as a new marker for depression in hospitalized heart failure patients.

Keywords: Neutrophilic granulocyte percentage, Depression, Hospitalized heart failure

\section{Background}

Heart failure is the end stage of all cardiovascular diseases, it mainly shows symptoms of dyspnea and edema, which severely affect exercise capacity and quality of life. Heart failure is prevalent around the globe, an estimated 5.7 million Americans have heart failure [1], and there are at

\footnotetext{
* Correspondence: wjk_sx@163.com

${ }^{\dagger}$ Equal contributors

${ }^{1}$ First Department of Cardiology, People's Hospital of Shaanxi Province, Xi'an 710068, Shaanxi, People's Republic of China

Full list of author information is available at the end of the article
}

least 15 million patients with heart failure in Europe [2]. Eleven million patients suffered from heart failure in the year 2010 in China [3]. Heart failure is one major cause of death and disability. Even though extensive pharmacologic and device therapeutics were developed for heart failure, the morbidity and mortality is still high in heart failure patients [4].

Depression is common in heart failure, with a reported prevalence of $21.5 \%$ in a meta-analysis [5]. However, the depression may be still under-diagnosed with the highest prevalence of up to $77.5 \%$ in one cohort study [6]. 
Depression is one of many factors associated with poor prognosis in heart failure patients [7-9]. It has been reported to be independently associated with poor quality of life, limited functional status, and increased risk of morbidity and mortality in this population [10-14].

Previous researches reveal that depression is associated with increased inflammatory markers including Creactive protein (CRP), interleukin-1, and interleukin-6 [15-17]. One study reports that the increased neutrophil/lymphocyte ratio is correlated with the severity of depression in patients with depression [18]. As one of the important indicator of inflammation, neutrophilic granulocyte percentage is cheap and simple test contained in blood routine test. We hypothesized that such an easily attained test result may be have certain association with depression and be used in predicting the depression in highly depressed heart failure patients. So far, the association between the neutrophilic granulocyte percentage and depression were not reported in such a population. Therefore, the aim of the present study is to investigate the association between the neutrophilic granulocyte percentage and depression in hospitalized heart failure patients.

\section{Methods}

\section{Subjects}

We performed this present cross-sectional and observational study in $1^{\text {st }}$ Cardiology Department of People's Hospital of Shaanxi Province from January 2015 to June in 2016. We enrolled hospitalized patients with left ventricular ejection fraction (LVEF) $\leq 45 \%$ and New York Heart Association (NYHA) class II-IV heart failure symptoms. New York Heart Association (NYHA) class II is defined as mild symptoms (mild shortness of breath and/or angina) and slight limitation during ordinary activity. New York Heart Association (NYHA) class III is defined as marked limitation in activity due to symptoms, even during less-than-ordinary activity, comfortable only at rest. New York Heart Association (NYHA) class IV is defined as severe limitations in activity, even while at rest, who are mostly bedbound patients [19]. Heart failure was confirmed by clinical heart failure specialist and cardiologists after consulting with the echocardiography report. The main exclusion criteria included significant cognitive impairment, alcohol or drug dependence within the previous year, psychoses, bipolar disorder, severe personality disorder, active suicidal ideation, life-threatening comorbidity, and current use of antipsychotic or antidepressant medications [20].

Three hundred eighty-five hospitalized patients with heart failure were screened, a total 19 patients were excluded. 8 persons were excluded as they were taking alprazolam and diazepam, 3 persons were excluded since they were heavy alcohol users, 4 persons were excluded as they had severe cognitive impairment after stroke, 2 persons were excluded as they suffered continuous dyspea and edema till death, 2 persons were excluded as they are not willing to cooperate. Among the enrolled 366 hospitalized patients, 200 (54.6\%) patients were male and $166(45.4 \%)$ patients were female. The average age was $62.56 \pm 10.74$ years.

\section{Depression assessments}

The heart failure patients were evaluated for depressive symptoms by using Hamilton Rating Scale for Depression (24-items) (HAM- $\left.{ }_{24}\right)$, it contained a total of 24 items (10 item was defined from 0 to 2 , and 14 items were defined from 0 to 4 ). Questions of $0-2$ points were defined as none (0), mild-moderate (1), severe (2). Questions of $0-4$ points were defined as none (0), mild (1), moderate (2), severe (3), very severe (4). HAM- $\mathrm{D}_{24}$ score $<8$ points was defined as non-depression and HAM- $\mathrm{D}_{24}$ score $\geq 8$ points was defined as depression. HAM- $\mathrm{D}_{24}$ score of 8-19 points was defined as mild depression, HAM- $\mathrm{D}_{24}$ score of $20-34$ points was defined as moderate depression, HAM- $\mathrm{D}_{24}$ score of $\geq 35$ points was defined as severe depression [21]. The measurements of HAM- $D_{24}$ were conducted by trained physicians on the first day after admission. Two physicians in our department were sent to psychology department for a week to learn the talking, observing and interpreting skill of HAM- $D_{24}$. The two physicians independently scores for each patients, the mean scores taken by the physicians were used as the final score. If the two scores were severely diverted, the repeated test will be performed by a psychologist in psychology department.

\section{Demographic and clinical data}

Demographic data and cardiovascular risk factors were obtained from the medical records. Body weight was measured while the subjects were without shoes by using a double balance placed on a firm surface. Height was measured by using a Frankfort plane positioned at a $90^{\circ}$ angle against a wall-mounted metal tape. The waist circumference measurements were taken at the end of normal expiration and to the nearest $0.1 \mathrm{~cm}$, measuring from the narrowest point between the lower borders of the rib cage and the iliac crest [22].

\section{Blood samples and echocardiography}

Peripheral blood was sampled from patients in a fasting state on the morning following the admission day. Venous blood samples were sent to Clinical Laboratory Department of People's Hospital of Shaanxi Province for red blood cells (RBC) counts, hemoglobin, platelet counts, plateletcrit, mean platelet volume (MPV), platelet distribution width (PDW), white blood cells (WBC) counts, neutrophilic granulocyte percentage (NEUT\%), 
total cholesterol (TC), triglyceride, high-density lipoproteincholesterol (HDL-C), low-density lipoprotein-cholesterol (LDL-C), apolipoprotein A, apolipoprotein B, urea nitrogen (BUN), creatinine, cystatin-C, total bilirubin (TBIL), direct bilirubin (DBIL), total protein (TP), albumin, brain natriuretic peptide (BNP) and fasting glucose (FG) detection using standard biochemical techniques. Echocardiographic data (left ventricular ejection fraction [LVEF]) was obtained using Doppler echocardiography conducted within 3 days of admission [23].

\section{Definition of risk factors}

Hypertension was defined as an average systolic blood pressure $\geq 140 \mathrm{mmHg}$, or an average diastolic blood pressure $\geq 90 \mathrm{mmHg}$, or both, or self-reported use of antihypertensive medication, or a self-reported history of hypertension.

Diabetes was defined as fasting plasma glucose $\geq$ $7.0 \mathrm{mmol} / \mathrm{L}$, or random plasma glucose $\geq 11.1 \mathrm{mmol} / \mathrm{L}$, or $2 \mathrm{~h}$ plasma glucose in oral glucose tolerance test $(\mathrm{OGTT}) \geq 11.1 \mathrm{mmol} / \mathrm{L}$, or use of insulin or oral hypoglycemic agents, or a self-reported history of diabetes.

Coronary artery disease was defined as the presence of at least one significant coronary artery stenosis of more than $50 \%$ luminal diameter in coronary angiography or coronary computed tomographic angiography (CTA).

Atrial fibrillation was diagnosed mainly via electrocardiogram (ECG), characteristic findings included absence of $P$ waves and irregular $R-R$ intervals.

Dilated cardiomyopathy was diagnosed mainly via echocardiogram, which showed left ventricular dilatation with normal or thinned walls and reduced ejection fraction. Meanwhile, the history of coronary artery disease, hypertension, valvular heart disease and other heart diseases should be excluded before making the present diagnosis.

Smoking index was defined as number of cigarettes smoked per day $\times$ years of smoking.

BMI was calculated as weight in $\mathrm{kg}$ divided by height in $\mathrm{m}^{2}$.

\section{Statistical analysis}

The statistical analysis was conducted using SPSS version 16.0 for Windows (SPSS Inc., Chicago, IL, USA). Continuous variables were expressed as mean \pm standard deviations and the differences between the depression group and non-depression group were analyzed using the Mann-Whitney $U$-test. Categorical variables were expressed as proportions and the differences in categorical variables were analyzed using chi-square test. Pearson correlation analysis was conducted to determine the correlation between HAM- $\mathrm{D}_{24}$ score and each clinical and laboratory factor. Logistic regression model was established to determine whether neutrophilic granulocyte percentage was significantly associated with depression after the adjustment of each confounding factor. Statistical significance was established at $p<0.05$.

\section{Results}

The baseline characteristics of hospitalized heart failure patients with and without depression were shown in Table 1. Two hundred ten patients developed depression accounting for $57.4 \%$ of all hospitalized patients with heart failure. Among them, 134 patients (63.8\%) had mild depression, 58 patients (27.6\%) had moderate depression and 18 patients (8.6\%) had severe depression. $31.3 \%$ of male patients had depression while $68.7 \%$ of female patients had depression, the distribution of depression between men and women was statistically significant. The distribution of depression in each New York Heart Association (NYHA) classification also varied significantly, the depression rates increased dramatically in patients with New York Heart Association (NYHA) class III and class IV. The incidence of coronary artery disease and diabetes mellitus showed significant differences between the two groups. The incidence of diabetes was higher in heart failure patients with depression while the incidence of coronary artery disease was lower in heart failure patients with depression when compared with that in heart failure patients without depression. The smoking index, platelet counts, plateletcrit, NEUT\%, TC, triglyceride, LDL-C, apolipoprotein B, BUN, creatinine, cystatin-C, BNP and HAM- ${ }_{24}$ score were significantly higher in heart failure patients with depression when compare with those without depression. Meanwhile, the waist circumference, monthly family income and hemoglobin and left ventricular ejection fraction (LVEF) were significantly lower in heart failure patients with depression when compare with those without depression. The habitant area, the incidence of hypertension, atrial fibrillation and dilated cardiomyopathy, age, BMI, number of members of the household, RBC counts, MPV, PDW, WBC counts, HDL-C, apolipoprotein A, TBIL, DBIL, TP, albumin and FG showed no significant difference between the heart failure patients with depression and ones without depression.

Pearson correlation analysis between HAM- $\mathrm{D}_{24}$ score and clinical and laboratory factors in hospitalized heart failure patients were shown in Table 2. The age, number of members of the household, smoking index, New York Heart Association (NYHA) classification, NEUT\%, TC, LDL-C, BUN, creatinine, cystatin-C, TBIL, DBIL and BNP showed significantly positive correlation with HAM$\mathrm{D}_{24}$ score in hospitalized heart failure patients. The correlation coefficient between NEUT\% and HAM- $\mathrm{D}_{24}$ score was $0.435(p<.001)$, which was the second highest correlation coefficient with $\mathrm{HAM}-\mathrm{D}_{24}$ in all the confounding 
Table 1 Baseline characteristics of hospitalized heart failure patients with and without depression

\begin{tabular}{|c|c|c|c|}
\hline & Without depression $(n=156)$ & With Depression $(n=210)$ & $p$ value \\
\hline Sex & & & $<.001$ \\
\hline Men & $104(52.0 \%)$ & $96(31.3 \%)$ & \\
\hline Women & $52(48.0 \%)$ & $114(68.7 \%)$ & \\
\hline Habitant area & & & .069 \\
\hline Urban area & $108(46.2 \%)$ & $48(36.4 \%)$ & \\
\hline Rural area & $126(53.8 \%)$ & $84(63.6 \%)$ & \\
\hline NYHA classification & & & $<.001$ \\
\hline$\|$ & $144(92.3 \%)$ & $122(58.1 \%)$ & \\
\hline III & $8(5.1 \%)$ & $68(32.4 \%)$ & \\
\hline IV & $4(2.6 \%)$ & $20(9.5 \%)$ & \\
\hline Coronary artery disease & $138(88.5 \%)$ & $164(78.1 \%)$ & .010 \\
\hline Hypertension & $90(57.7 \%)$ & $116(55.2 \%)$ & .640 \\
\hline Atrial fibrillation & $26(16.7 \%)$ & $48(22.9 \%)$ & .145 \\
\hline Dilated cardiomyopathy & $8(5.1 \%)$ & $18(8.6 \%)$ & .205 \\
\hline Diabetes mellitus & $42(26.9 \%)$ & 78 (37.1\%) & .039 \\
\hline Age (years) & $62.55 \pm 9.56$ & $62.58 \pm 11.61$ & .723 \\
\hline BMI $\left(\mathrm{kg} / \mathrm{m}^{2}\right)$ & $23.82 \pm 2.73$ & $23.46 \pm 4.33$ & .404 \\
\hline Waist circumference (cm) & $86.18 \pm 8.71$ & $84.16 \pm 10.29$ & .017 \\
\hline Monthly family income (yuan) & $3869.23 \pm 3118.49$ & $3019.05 \pm 1782.27$ & .003 \\
\hline Number of members of the household (person) & $4.04 \pm 1.27$ & $3.88 \pm 1.35$ & .199 \\
\hline Smoking index & $73.97 \pm 203.39$ & $100.14 \pm 190.25$ & $<.001$ \\
\hline RBC counts $\left(\times 10^{12} / \mathrm{L}\right)$ & $4.35 \pm 0.49$ & $4.21 \pm 0.60$ & .091 \\
\hline Hemoglobin (g/L) & $135.24 \pm 16.05$ & $131.00 \pm 18.37$ & .035 \\
\hline Platelet counts $\left(\times 10^{9} / \mathrm{L}\right)$ & $176.54 \pm 45.62$ & $190.26 \pm 58.96$ & .018 \\
\hline Plateletcrit (\%) & $0.18 \pm 0.05$ & $0.19 \pm 0.05$ & .021 \\
\hline MPV (fL) & $10.33 \pm 1.48$ & $10.30 \pm 1.31$ & .954 \\
\hline PDW (fL) & $16.45 \pm 2.28$ & $16.24 \pm 2.05$ & .127 \\
\hline WBC counts $\left(\times 10^{9} / \mathrm{L}\right)$ & $6.93 \pm 5.00$ & $6.54 \pm 1.82$ & .285 \\
\hline NEUT\% (\%) & $30.69 \pm 32.60$ & $63.31 \pm 12.13$ & $<.001$ \\
\hline $\mathrm{TC}(\mathrm{mmol} / \mathrm{L})$ & $3.95 \pm 0.88$ & $4.67 \pm 1.13$ & $<.001$ \\
\hline Triglyceride (mmol/L) & $1.40 \pm 0.69$ & $1.76 \pm 1.18$ & .015 \\
\hline HDL-C (mmol/L) & $1.19 \pm 0.28$ & $1.18 \pm 0.36$ & .390 \\
\hline LDL-C (mmol/L) & $1.87 \pm 0.60$ & $2.26 \pm 0.77$ & $<.001$ \\
\hline Apolipoprotein A (g/L) & $1.14 \pm 0.21$ & $1.14 \pm 0.27$ & .748 \\
\hline Apolipoprotein B (g/L) & $0.72 \pm 0.19$ & $1.22 \pm 3.47$ & $<.001$ \\
\hline BUN (mmol/L) & $5.82 \pm 2.51$ & $6.65 \pm 3.50$ & .046 \\
\hline Creatinine (umol/L) & $73.98 \pm 24.26$ & $85.63 \pm 40.81$ & .001 \\
\hline Cystatin-C (mg/L) & $1.09 \pm 0.32$ & $1.27 \pm 0.54$ & .001 \\
\hline TBIL (umol/L) & $17.12 \pm 8.90$ & $18.63 \pm 15.39$ & .913 \\
\hline DBIL (umol/L) & $5.94 \pm 3.13$ & $6.67 \pm 6.94$ & .410 \\
\hline TP (g/L) & $64.04 \pm 5.70$ & $63.08 \pm 6.15$ & .134 \\
\hline Albumin (g/L) & $38.34 \pm 5.47$ & $37.51 \pm 4.91$ & .343 \\
\hline BNP (pg/mL) & $364.15 \pm 832.65$ & $601.65 \pm 986.63$ & $<.001$ \\
\hline
\end{tabular}


Table 1 Baseline characteristics of hospitalized heart failure patients with and without depression (Continued)

\begin{tabular}{llll}
\hline FG $(\mathrm{mmol} / \mathrm{L})$ & $6.63 \pm 1.54$ & $7.66 \pm 2.78$ & .148 \\
LVEF (\%) & $39.29 \pm 3.25$ & $35.59 \pm 6.94$ & $<.001$ \\
HAM-D & & & \\
H Score & $4.32 \pm 1.67$ & $17.58 \pm 8.36$ & $<.001$ \\
\hline
\end{tabular}

NYHA New York Heart Association, BMI body mass index, RBC red blood cells, MPV mean platelet volume, PDW platelet distribution width, WBC white blood cells, NEUT\% neutrophilic granulocyte percentage, TC total cholesterol, HDL-C high-density lipoprotein-cholesterol, LDL-C low-density lipoprotein-cholesterol, BUN urea nitrogen, TBIL total bilirubin, DBIL direct bilirubin, $T P$ total protein, $B N P$ brain natriuretic peptide, FG fasting glucose, $L V E F$ left ventricular ejection fraction

Table 2 Pearson correlation analysis between HAM-D 24 score and clinical and laboratory factors in hospitalized heart failure patients

\begin{tabular}{|c|c|c|}
\hline & $r$ & $p$ value \\
\hline Age & .104 & $.046^{*}$ \\
\hline BMl & -.106 & $.043^{*}$ \\
\hline Waist circumference & -.076 & .149 \\
\hline Monthly family income & -.076 & .147 \\
\hline Number of members of the household & .143 & $.006^{*}$ \\
\hline Smoking index & .103 & $.048^{*}$ \\
\hline NYHA classification & .603 & $<.001^{*}$ \\
\hline RBC counts & -.189 & $<.001^{*}$ \\
\hline Hemoglobin & -.146 & $.007^{*}$ \\
\hline Platelet counts & -.044 & .420 \\
\hline Plateletcrit & -.049 & .369 \\
\hline MPV & .009 & .864 \\
\hline PDW & .019 & .730 \\
\hline WBC counts & -.097 & .074 \\
\hline NEUT\% & .435 & $<.001^{*}$ \\
\hline TC & .326 & $<.001^{*}$ \\
\hline Triglyceride & .057 & .286 \\
\hline $\mathrm{HDL}-\mathrm{C}$ & -.053 & .328 \\
\hline LDL-C & .354 & $<.001^{*}$ \\
\hline Apolipoprotein A & -.089 & .099 \\
\hline Apolipoprotein B & .008 & .878 \\
\hline BUN & .162 & $.003^{*}$ \\
\hline Creatinine & .190 & $<.001^{*}$ \\
\hline Cystatin-C & .269 & $<.001^{*}$ \\
\hline TBIL & .150 & $.005^{*}$ \\
\hline DBIL & .137 & $.011^{*}$ \\
\hline TP & -.068 & .210 \\
\hline Albumin & -.175 & $.001^{*}$ \\
\hline BNP & .243 & $<.001^{*}$ \\
\hline FG & .090 & .524 \\
\hline LVEF & -.271 & $<.001^{*}$ \\
\hline
\end{tabular}

$H A M-D_{24}$ Hamilton rating scale for depression (24 items), $B M I$ body mass index, NYHA New York Heart Association, RBC red blood Cells, MPV mean platelet volume, $P D W$ platelet distribution width, WBC white blood cells, NEUT\% neutrophilic granulocyte percentage, TC total cholesterol, HDL-C high-density lipoproteincholesterol, LDL-C low-density lipoprotein-cholesterol, BUN urea nitrogen, TBIL total bilirubin, DBIL direct bilirubin, $T P$ total protein, $B N P$ brain natriuretic peptide, FG fasting glucose, LVEF left ventricular ejection fraction; ${ }^{*} P<0.05$ factors. Meanwhile, the BMI, RBC counts, hemoglobin, albumin and left ventricular ejection fraction (LVEF) showed significantly negative correlation with HAM- $\mathrm{D}_{24}$ score in hospitalized heart failure patients. The waist circumference, monthly family income, platelet counts, plateletcrit, MPV, PDW, WBC counts, triglyceride, HDL-C, apolipoprotein A, apolipoprotein B, TP, FG showed no significant correlation with HAM- $\mathrm{D}_{24}$ score in hospitalized heart failure patients.

Logistic regression analysis for $\mathrm{HAM}-\mathrm{D}_{24}$ determined depression using neutrophilic granulocyte percentage and each confounding factor in hospitalized heart failure patients were presented in Table 3. We enrolled all the factors significantly correlated with $\mathrm{HAM}-\mathrm{D}_{24}$ score except for RBC counts, BUN, DBIL, BNP and left ventricular ejection fraction (LVEF) in the Logistic regression. Since the highly tight correlations were found between hemoglobin and RBC counts, BUN and creatinine, DBIL and TBIL, New York Heart Association (NYHA) classification and BNP, left ventricular ejection fraction (LVEF) in clinical practices, we considered it inappropriate if we put all the highly correlated factors in one Logistic regression model. Therefore, we chose the hemoglobin, creatinine, TBIL and New York Heart Association (NYHA) classification in the Logistic regression model rather than RBC counts, BUN, DBIL, BNP and left ventricular ejection fraction (LVEF). After the adjustment of age, BMI, number of members of the household, smoking index, New York Heart Association (NYHA) classification, hemoglobin, TC, LDL-C, creatinine, cystatin-C, TBIL and albumin, NEUT\% is still significantly associated with depression in hospitalized heart failure patients $(O R=1.046, p<.001)$.

\section{Discussions}

The present study revealed that correlation coefficient between NEUT\% and HAM-D ${ }_{24}$ score was $0.435(p<.001)$. Then the Logistic regression model confirmed that NEUT\% is still associated with depression in hospitalized heart failure patients with the adjustment of age, BMI, number of members of the household, smoking index, New York Heart Association (NYHA) classification, hemoglobin, TC, LDL-C, creatinine, cystatin-C, TBIL and albumin $(O R=1.046, p<.001)$. The mechanism that the NEUT\% was associated with depression in heart failure patients is still unclear. The possible reason may be 
Table 3 Logistic regression analysis for HAM- $\mathrm{D}_{24}$ determined depression using neutrophilic granulocyte percentage and each confounding factor in hospitalized heart failure patients

\begin{tabular}{llll}
\hline & Odd ratios & $95 \%$ Cl & $p$ value \\
\hline Age & 0.988 & $0.959-1.018$ & .420 \\
BMl & 0.996 & $0.914-1.086$ & .933 \\
Number of members of the household & 0.945 & $0.754-1.184$ & .622 \\
Smoking index & 1.000 & $0.998-1.002$ & .830 \\
NYHA classification & 2.757 & $1.361-5.586$ & $.005^{*}$ \\
Hemoglobin & 0.987 & $0.967-1.007$ & .191 \\
NEUT\% & 1.046 & $1.029-1.063$ & $<.001^{*}$ \\
TC & 2.038 & $1.213-3.424$ & $.007^{*}$ \\
LDL-C & 0.942 & $0.459-1.933$ & .871 \\
Creatinine & 0.996 & $0.976-1.016$ & .703 \\
Cystatin-C & 1.589 & $0.420-6.017$ & .495 \\
TBIL & 0.994 & $0.966-1.023$ & .693 \\
Albumin & 0.952 & $0.897-1.011$ & .111 \\
\hline
\end{tabular}

$H A M-D_{24}$ Hamilton rating scale for depression (24 items), Cl confidence interval, BMI body mass index, NYHA New York Heart Association, NEUT\% neutrophilic granulocyte percentage, $T C$ total cholesterol, $L D L-C$ low-density lipoprotein-cholesterol, $T B I L$ total bilirubin; ${ }^{*} P<0.05$

as follows. As an important indicator for inflammation, the neutrophilic granulocytes would be activated firstly when responding to inflammation exposure. The activated neutrophilic granulocytes, which can be interpreted as increased NEUT\% may be accompanied with elevated inflammatory cytokines secretion causing heavier oxidative stress and further inflammation. Meanwhile, some studies [24, 25] found that in patients with severe depressive disorder, the activation of proinflammatory cytokines and inhibition of interferon-gamma, interleukin-2, and interleukin- 4 were documented. Therefore, we believe that the activation of inflammation may play the key role in the association with depression. The heart failure may amplify the association via the increase of inflammation and oxidative stress. However, the causal relationship between the inflammation and depression needs to be further clarified.

The present study showed that the depression rates increased dramatically in patients with New York Heart Association (NYHA) class III and class IV, we also found that the predictors of severity of heart failure, such as New York Heart Association (NYHA) classification, BNP, and EF were all significantly correlated with HAM$\mathrm{D}_{24}$ score (all $p<.001$ ). The correlation coefficients were 0.603 for New York Heart Association (NYHA) classification, -0.271 for left ventricular ejection fraction (LVEF) and 0.243 for BNP, the New York Heart Association (NYHA) classification was proved to be a risk factor after adjustment of other confounding factors $(O R=2.757, p=.005)$. Relevant research has also shown a higher prevalence of depression in patients with more severe heart failure [26, 27]. Pena et al. [28] showed that heart failure patients in New York Heart Association
(NYHA) grade IV were more depressed than those in New York Heart Association (NYHA) grades II or III. Gottlieb et al. [6] demonstrated that patients classified as New York Heart Association (NYHA) class III and IV were more likely to be depressed than class II patients. In their meta-analysis, Rutledge et al. [5] demonstrated that higher prevalencerates were associated with worse New York Heart Association (NYHA) class. Polikandrioti et al. [4] found that higher levels of depression were observed for heart failure patients in New York Heart Association (NYHA) II and NYHA III compared to those in grade I.

In this present study, we found that the traditional risk factors for heart failure were significantly correlated with HAM-D ${ }_{24}$ score. A part of the reason may be that anemia (counts and hemoglobin), dyslipidemia (TC and LDL-C), kidney dysfunction (BUN, creatinine and cystatin-C) and liver dysfunction (TBIL, DBIL and albumin) were classical features accompanied with heart function deterioration, as the heart function decreased, the symptoms of heart failure began to appear, which may cause the depression in this population [29-32]. Besides these traditional factors, the present study revealed 4 demographic factors significantly correlated with HAM-D ${ }_{24}$ score. First, HAM-D ${ }_{24}$ score increased as patients got elder, considering that the cardiovascular diseases were highly age related, it is not difficult to understand that depression level will rise with increase of age. Second, BMI would decrease as the increase of depression level, a majority of heart failure patients had sodium and water retention, remission of sodium and water retention will lead to some weight loss, which will improve symptoms of heart failure and depression [33]. Third, larger number of members of the household was 
correlated with more depression. This was an interesting Chinese phenomenon that the more number of members of the household did not mean more family support and less loneliness, it meant more argument and relatively less space in the room for each person. Fourth, smoking index significantly increased as HAM-D ${ }_{24}$ score increased. It was also an interesting phenomenon that smoking may not necessarily bring you happiness and relief, it made you more depressed if you had heart failure. The reason may be that smoking activated the process of arteriosclerosis making you more vulnerable to the harm of cardiovascular disease [34].

By using HAM- $\mathrm{D}_{24}$ score, a convenient and accurate tool for the depression evaluation $[35,36]$, the present study showed that $57.4 \%$ of all hospitalized patients with heart failure had depression. The prevalence of depression in heart failure patients varied dramatically in previous studies. Rutledge et al. [5] reported the prevalence of depression as $21.5 \%$ in a meta-analytic review. Rafanelli et al. [37] who studied both hospitalized patients and outpatients with heart failure, showed that $38.1 \%$ of the sample experienced depression. One study in Greek population [38] showed that $41.6 \%$ of hospitalized patients with heart failure appeared to have depression. Gottlieb et al. [6] reported depression rates among hospitalized patients of 13 to $77.5 \%$ and rates for outpatients from 13 to $42 \%$. Vaccarino et al. [39] showed that $78 \%$ of hospitalized heart failure patients experienced depressive symptoms. These differences in the prevalence of depression in heart failure patients are possibly attributable to differences in enrollment criteria, diagnostic instruments, the definition and classification of depression, and the severity of heart failure. This highlights the importance of using a universally accepted measuring instrument that will allow comparisons between populations.

Our study has several strengths. First, we provide clinical and laboratory data in hospitalized heart failure patients divided by depression and the relationship between the confounding factors and depression, which conveyed valuable clue for other studies searching new risk factors for depression in hospitalized heart failure patients. Second, we have shown for the first time the association between NEUT\% and depression in hospitalized heart failure patients. Third, the blood routine test is a simple, cheap and commonly used test, so NEUT\% can be easily acquired. NEUT\% would present us with new insight into the depression status in hospitalized heart failure patients.

Our study has several limitations. This study was a cross-sectional and observational study, the causal relationship between NEUT\% and depression need to be further clarified. Further cohort studies may provide treatment to patients with depression and then evaluate whether the NEUT\% showed the decrease trend after the depression was alleviated in this population. Since the patients with severe and very severe level depression were limited, we did not further divide our patients into different groups according to severity of depression. Therefore, further larger scale, prospective studies are needed to explore extensive information in patients divided according to severity of depression. Third, we used the self-reported history of hypertension and diabetes in the paper, which may underestimate the actual incidence of hypertension and diabetes in the enrolled patients. Fourth, according to Bonferroni correction, the significant of $\mathrm{TC}$ as a risk factor for depression should be further considered since the $p$ value should be divided by 13 (13 independent factors) in logistic regression analysis.

\section{Conclusions}

In conclusion, NEUT\% was significantly and positively correlated with HAM- $\mathrm{D}_{24}$ score in hospitalized heart failure patients. Moreover, after the adjustment of confounding factors, the NEUT\% is independently associated with depression, it can be used as an new marker for depression in hospitalized heart failure patients. In the future, a simple and cheap NEUT\% test may provide insight into depression status and should be included in psychiatric evaluation in hospitalized heart failure patients.

\section{Abbreviations \\ BMI: Body mass index; BNP: Brain natriuretic peptide; BUN: Urea nitrogen; \\ $\mathrm{Cl}$ : Confidence interval; DBIL: Direct bilirubin; FG: Fasting glucose; HAM- \\ $\mathrm{D}_{24}$ : Hamilton rating scale for depression (24 items); HDL-C: High-density lipoprotein-cholesterol; LDL-C: Low-density lipoprotein-cholesterol; LVEF: Left ventricular ejection fraction; MPV: Mean platelet volume; NEUT\%: Neutrophilic granulocyte percentage; NYHA: New York heart association; PDW: Platelet distribution width; RBC: Red blood cells; TBIL: Total bilirubin; TC: Total cholesterol; TP: Total protein; WBC: White blood cells}

\section{Acknowledgements}

This paper was funded by National Natural Science Funds of China (Grant No. 81500308)

\section{Funding}

This paper was funded by National Natural Science Funds of China (Grant No. 81500308).

\section{Availability of data and materials}

All data generated or analysed during this study are included in this published article. The datasets generated during and/or analysed during the current study are not publicly available due to participants' rights to privacy, but they are available from the corresponding author on reasonable request.

\section{Authors' contributions}

Conceived and designed the experiments: SP, Z-WL, YL, J-KW. Performed the experiments: W-QS, XM, G-CG, YZ, S-MZ, F-QL, BL, Z-GT. Analyzed the data: SP Z-WL. Contributed reagents/materials/analysis tools: SP, Z-WL, J-KW. Wrote the manuscript: SP, J-KW. All authors read and approved the final manuscript.

Competing interests

The authors declare that they have no competing interests.

Consent for publication

The consents to publish the paper were obtained from all authors. 


\section{Ethics approval and consent to participate}

This study was approved by the Ethics Committee of the People's Hospital of Shaanxi Province (Xi'an, China). Written informed consent was obtained from all participants. We have obtained consent to publish from the participants to report individual patient data. It was conducted according to the standards of the Declaration of Helsinki.

\section{Author details}

'First Department of Cardiology, People's Hospital of Shaanxi Province, Xi'an 710068, Shaanxi, People's Republic of China. ${ }^{2}$ Department of Internal Medicine, University Hospital of Northwest University, Xi'an, Shaanxi, People's Republic of China. ${ }^{3}$ Department of Emergency Medicine, People's Hospital of Shaanxi Province, Xi'an, Shaanxi, People's Republic of China.

\section{Received: 2 August 2016 Accepted: 7 December 2016} Published online: 13 December 2016

\section{References}

1. Lloyd-Jones D, Adams R, Carnethon M, De Simone G, Ferguson TB, Flegal K, et al. Heart disease and stroke statistics-2009 update: a report from the American heart association statistics committee and stroke statistics subcommittee. Circulation. 2009;119(3):480-6.

2. Dickstein K, Cohen-Solal A, Filippatos G, McMurray JJ, Ponikowski P, PooleWilson PA, et al. ESC guidelines for the diagnosis and treatment of acute and chronic heart failure 2008: the task force for the diagnosis and treatment of acute and chronic heart failure 2008 of the European society of cardiology. Developed in collaboration with the heart failure association of the ESC (HFA) and endorsed by the European society of intensive care medicine (ESICM). Eur Heart J. 2008;29(19):2388-442.

3. Zhou J, Cui X, Ge J. The epidemiological profile of heart failure patients in China. Zhonghua Xin Xue Guan Bing Za Zhi. 2015;43(12):1018-21.

4. Hunt SA, Abraham WT, Chin MH, Feldman AM, Francis GS, Ganiats TG, et al. ACC/AHA 2005 guideline update for the diagnosis and management of chronic heart failure in the adult: a report of the American college of cardiology/American heart association task force on practice guidelines (writing committee to update the 2001 guidelines for the evaluation and management of heart failure): developed in collaboration with the American college of chest physicians and the international society for heart and lung transplantation: endorsed by the heart rhythm society. Circulation. 2005;112(12):e154-235.

5. Rutledge T, Reis VA, Linke SE, Greenberg BH, Mills PJ. Depression in heart failure a metaanalytic review of prevalence, intervention effects, and associations with clinical outcomes. J Am Coll Cardiol. 2006;48(8):1527-37.

6. Gottlieb SS, Khatta M, Friedmann E, Einbinder L, Katzen S, Baker B, et al. The influence of age, gender, and race on the prevalence of depression in heart failure patients. J Am Coll Cardiol. 2004;43(9):1542-9.

7. Lee DS, Austin PC, Rouleau JL, Liu PP, Naimark D, Tu JV. Predicting mortality among patients hospitalized for heart failure: derivation and validation of a clinical model. JAMA. 2003;290(19):2581-7.

8. Levy WC, Mozaffarian D, Linker DT, Sutradhar SC, Anker SD, Cropp AB, et al. The seattle heart failure model: prediction of survival in heart failure. Circulation. 2006:113:1424-33.

9. Levy WC, Linker DT. Prediction of mortality in patients with heart failure and systolic dysfunction. Curr Cardiol Rep. 2008;10(3):198-205

10. Jiang W, Kuchibhatla M, Clary GL, Cuffe MS, Christopher EJ, Alexander JD, et al. Relationship between depressive symptoms and long-term mortality in patients with heart failure. Am Heart J. 2007;154(1):102-8.

11. Johansson P, Dahlstrom U, Alehagen U. Depressive symptoms and six-year cardiovascular mortality in elderly patients with and without heart failure. Scand Cardiovasc J. 2007:41(5):299-307.

12. Muller-Tasch T, Peters-Klimm F, Schellberg D, et al. Depression is a major determinant of quality of life in patients with chronic systolic heart failure in general practice. J Card Fail. 2007;13(10):818-24.

13. O'Connor CM, Jiang W, Kuchibhatla M, Mehta RH, Clary GL, Cuffe MS, et al. Antidepressant use, depression, and survival in patients with heart failure. Arch Intern Med. 2008;168(20):2232-7.

14. Gottlieb SS, Kop WJ, Ellis SJ, Binkley P, Howlett J, O'Connor C, et al. Relation of depression to severity of illness in heart failure (from Heart Failure And a Controlled Trial Investigating Outcomes of Exercise Training [HFACTION]). Am J Cardiol. 2009;103(9):1285-9.
15. Dekker RL, Moser DK, Tovar EG, Chung ML, Heo S, Wu JR, et al. Depressive symptoms and inflammatory biomarkers in patients with heart failure. Eur J Cadiovasc Nurs. 2014;13(5):444-50.

16. Elovainio $M$, Aalto AM, Kivimäki M, Pirkola S, Sundvall J, Lönnqvist J, et al. Depression and C-reactive protein: population-based Health 2000 study. Psychosom Med. 2009;71(4):423-30.

17. Wium-Andersen MK, Orsted DD, Nordestgaard BG. Elevated C-reactive protein, depression, somatic diseases, and all-cause mortality: a mendelian randomization study. Biol Psychiatry. 2014;76(3):249-57.

18. Aydin Sunbul E, Sunbul M, Yanartas O, Cengiz F, Bozbay M, Sari I, et al. Increased neutrophil/lymphocyte ratio in patients with depression is correlated with the severity of depression and cardiovascular risk factors. Psychiatry Investig. 2016;13(1):121-6.

19. Ahmed A. American college of cardiology/American heart association chronic heart failure evaluation and management guidelines: relevance to the geriatric practice. J Am Geriatr Soc. 2003;51(1):123-6.

20. O'Connor CM, Jiang W, Kuchibhatla M, Silva SG, Cuffe MS, Callwood DD, et al, Safety and efficacy of sertraline for depression in patients with heart failure: results of the SADHART-CHF (Sertraline Against Depression and Heart Disease in Chronic Heart Failure) trial. J Am Coll Cardiol. 2010;56(9):692-9.

21. Wang L, Zhong Z, Hu J, Rong X, Liu J, Xiao S, et al. Sertraline plus deanxit to treat patients with depression and anxiety in chronic somatic diseases: a randomized controlled trial. BMC Psychiatry. 2015;15:84.

22. Pan S, Yu ZX, Ma YT, Liu F, Yang YN, Ma X, et al. Appropriate body mass index and waist circumference cutoffs for categorization of overweight and central adiposity among Uighur adults in Xinjiang. PLoS One. 2013;8(11):e80185.

23. Zhao N, Mi L, Liu X, Pan S, Xu J, Xia D, et al. Combined value of red blood cell distribution width and global registry of acute coronary events risk score for predicting cardiovascular events in patients with acute coronary syndrome undergoing percutaneous coronary intervention. PLoS One. 2015; 10(10):e0140532.

24. Kasama T, Miwa Y, Isozaki T, Odai T, Adachi M, Kunkel SL. Neutrophil-derived cytokines: potential therapeutic targets in inflammation. Curr Drug Targets Inflamm Allergy. 2005;4(3):273-9.

25. Kim YK, Na KS, Shin KH, Jung HY, Choi SH, Kim JB. Cytokine imbalance in the pathophysiology of major depressive disorder. Prog Neuropsychopharmacol Biol Psychiatry. 2007;31(5):1044-53.

26. Carels RA. The association between disease severity, functional status, depression and daily quality of life in congestive heart failure patients. Qual Life Res. 2004;13(1):63-72.

27. Faller H, Störk S, Schuler M, Schowalter M, Steinbüchel T, Ertl G, et al. Depression and disease severity as predictors of health-related quality of life in patients with chronic heart failure-a structural equation modeling approach. J Card Fail. 2009:15(4):286-292.e2.

28. Pena FM, da Silva SJ, Paiva BT, Piraciaba MC, Marins RM, Barcellos AF, et al. Sociodemographic factors and depressive symptoms in hospitalized patients with heart failure. Exp Clin Cardiol. 2010;15(2):e29-32.

29. Vulser H, Wiernik E, Hoertel N, Thomas F, Pannier B, Czernichow S, et al. Association between depression and anemia in otherwise healthy adults. Acta Psychiatr Scand. 2016;134(2):150-60.

30. van Reedt Dortland AK, Vreeburg SA, Giltay E, Licht CM, Vogelzangs N, van Veen $\mathrm{T}$, et al. The impact of stress systems and lifestyle on dyslipidemia and obesity in anxiety and depression. Psychoneuroendocrinology. 2013;38(2):209-18.

31. Bautovich A, Katz I, Smith M, Loo CK, Harvey SB. Depression and chronic kidney disease: a review for clinicians. Aust N Z J Psychiatry. 2014;48(6):530-41.

32. Schafer K, Butters N, Smith T, Irwin M, Brown S, Hanger P, et al. Cognitive performance of alcoholics: a longitudinal evaluation of the role of drinking history, depression, liver function, nutrition, and family history. Alcohol Clin Exp Res. 1991;15(4):653-60.

33. Han SS, Bae E, Kim DK, Kim YS, Han JS, Joo KW. Dysnatremia, its correction, and mortality in patients undergoing continuous renal replacement therapy: a prospective observational study. BMC Nephrol. 2016;17:2.

34. Knowles S, Planner C, Bradshaw T, Peckham E, Man MS, Gilbody S. Making the journey with me: a qualitative study of experiences of a bespoke mental health smoking cessation intervention for service users with serious mental illness. BMC Psychiatry. 2016;16:193.

35. Montoya A, Lebrec J, Keane KM, Fregenal I, Ciudad A, Moríñigo A, et al. Broader conceptualization of remission assessed by the remission from depression questionnaire and its association with symptomatic remission: a prospective, multicenter, observational study. BMC Psychiatry. 2016;16(1):352. 
36. Choi NG, Marti CN, Bruce ML, Kunik ME. Relationship between depressive symptom severity and emergency department use among low-income, depressed homebound older adults aged 50 years and older. BMC Psychiatry. 2012:12:233.

37. Rafanelli C, Gostoli S, Tully PJ, Roncuzzi R. Hostility and the clinical course of outpatients with congestive heart failure. Psychol Health. 2016;31(2):228-38.

38. Polikandrioti M, Goudevenos J, Michalis LK, Koutelekos J, Kyristi H, Tzialas D, et al. Hostility and the clinical course of outpatients with congestive heart failure. Psychol Health. 2016;31(2):228-38.

39. Vaccarino V, Kasl SV, Abramson J, Krumholz HM. Depressive symptoms and risk of functional decline and death in patients with heart failure. J Am Coll Cardiol. 2001;38(1):199-205.

Submit your next manuscript to BioMed Central and we will help you at every step:

- We accept pre-submission inquiries

- Our selector tool helps you to find the most relevant journal

- We provide round the clock customer support

- Convenient online submission

- Thorough peer review

- Inclusion in PubMed and all major indexing services

- Maximum visibility for your research

Submit your manuscript at www.biomedcentral.com/submit
Biomed Central 